\title{
The role of predictability in preventing escape deficits following loss of control over food acquisition
}

\author{
AKIHITO SONODA and HISASHI HIRAI \\ Sophia University, Tokyo, Japan
}

\begin{abstract}
The present experiment examined whether predictability of food acquisition would eliminate the impairment of subsequent escape performance that otherwise resulted from uncontrollability over food acquisition. In pretreatment, the yoked and the yoked-signal groups received responseindependent food at the same times as the experimental group acquired it on an FR 5/20 leverpress schedule. However, a pellet presented for the yoked-signal group followed a 1.5-sec tone, which served as a predictive signal of food. The naive control group received the same number of pellets in their home cages in this phase. Results of the escape latency in the subsequent FR 2 shuttling shock-escape test indicated that the predictability of outcome eliminated the escape deficits showed by the yoked-nonsignal group. This modulating effect of a predictive signal is hypothesized to be due to an overshadowing of uncontrollability by predictability.
\end{abstract}

Exposure to inescapable shock is known to interfere with later adaptive learning (Overmier \& Seligman, 1967; Seligman \& Maier, 1967). This phenomenon has been termed the learned helplessness effect; it has been hypothesized that learning about noncontingency between response and outcome produces the learning of uncontrollability and results in deleterious effects (Maier \& Seligman, 1976; Seligman, 1975). Findings that the learning of uncontrollability in an appetitive situation interferes with subsequent learning (Caspy \& Lubow, 1981; Goodkin, 1976; Job, 1987, 1988, 1989; Sonoda, Hirai, \& Okayasu, in press; Sonoda, Okayasu, \& Hirai, 1991) are formally similar to effects seen with uncontrollability over shock. Both effects of aversive and appetitive pretreatment appear to result from the learning of response-outcome independence and seem to support the importance of control over outcomes.

To date, much literature has reported that the presence of an exteroceptive stimulus ameliorated the adverse effects of inescapable shocks when such a stimulus was used as a feedback (backward) stimulus following shock (e.g., DeCola, Rosellini, \& Warren, 1988; Maier \& Warren, 1988; Mineka, Cook, \& Miller, 1984; Rosellini, Warren, \& DeCola, 1987; Volpicelli, Ulm, \& Altenor, 1984), a cessation signal of shock (Minor, Trauner, Lee, \& Dess,

This work was supported by Grant-in-Aid for Scientific Research (JSPS Fellowships for the Japanese Junior Scientists) to the first author. We wish to express our thanks to Akira Tsuda of the Kurume University School of Medicine and Takahiro Okayasu of Waseda University for their invaluable advice and encouragement. We also wish to express our thanks to Gary B. Glavin of the University of Manitoba for his review of an earlier version of this manuscript. Requests for reprints should be directed to Akihito Sonoda, Department of Psychology, Sophia University, Tokyo 102, Japan.
1990), or a forward stimulus followed by shock (predictable shock) (e.g., Dess, Linwick, Patterson, Overmier, \& Levine, 1983; Jackson \& Minor, 1988; Seligman, 1968; Seligman \& Meyer, 1970). However, little is known about whether the interaction of control and prediction in appetitive situations influences subsequent performance. The purpose of the present experiment is to examine whether predictability of food acquisition eliminates the deficits typically observed in shock-escape test situations following loss of control over food acquisition (Sonoda et al., 1991, in press).

\section{METHOD}

\section{Subjects}

Thirty-six naive male Wistar-strain rats, about 9 weeks old (each weighing approximately $250 \mathrm{~g}$ at the beginning of the study), were used as subjects. All animals were housed in a temperature- $\left(25^{\circ} \mathrm{C}\right)$ and humidity- (approximately $45 \%$ ) regulated animal room, in groups of 2-3 rats per cage. They were maintained on a 12:12-h light:dark cycle with water available ad lib.

\section{Apparatus}

Three identical Skinner boxes and a shuttle box were used. The Skinner boxes used in continuous reinforcement (CRF) training and pretreatment consisted of a chamber with clear Plexiglas sidewalls and ceiling, stainless steel front and rear walls, and a floor of stainless steel rods $(1.6 \mathrm{~cm}$ center to center). The internal dimensions were $23.5 \mathrm{~cm}$ wide $\times 25.5 \mathrm{~cm}$ high $\times 30.0 \mathrm{~cm}$ long. The front wall had an attached food cup, $1.0 \mathrm{~cm}$ in diameter, located $2.5 \mathrm{~cm}$ above the floor and $12.5 \mathrm{~cm}$ from the right sidewall. A $2-\mathrm{cm}$ long, $3-\mathrm{cm}$ wide stainless steel bar was located $4.0 \mathrm{~cm}$ from the right sidewall, $5.5 \mathrm{~cm}$ above the floor. Leverpresses were automatically detected and recorded by a personal computer (PC-8801 or PC9801), and 45-mg food pellets (Holton Industries) were delivered by a pellet dispenser. A speaker was attached to the outside of the front wall of each Skinner box. The auditory signal ( $90 \mathrm{~dB}$, $1000 \mathrm{~Hz}$ ) originated from an oscillator and was delivered through the speaker. 
The escape test was administered in a $20-\mathrm{cm}$ wide $\times 20$-cm high $\times 46-\mathrm{cm}$ long two-way shuttle box consisting of clear Plexiglas sidewalls and stainless steel endwalls and a grid floor $(1.1 \mathrm{~cm}$ center to center). The center of the shuttle box contained a metal divider with a rounded archway $9.5 \mathrm{~cm}$ high and $6.3 \mathrm{~cm}$ wide cut out of it. Scrambled shocks $(0.8 \mathrm{~mA})$ were delivered to the grid floors by a SGS-001 shock generator/scrambler (Muromachi Kikai Co.).

\section{Procedure}

The animals were placed on a 23-h food-deprivation schedule, with access to food during $1 \mathrm{~h}$ per day, over a 5-day period prior to the experimental session. This reduced their weight by approximately $15 \%$ prior to the beginning of the experiment.

Shaping and CRF training. On the first day, 27 animals received shaping of leverpress responses in a Skinner box with about 50 food pellets. On the following day, these rats were administered one CRF training session to earn 100 pellets by leverpressing in the Skinner box. The remaining 9 animals consumed 50 pellets on the first day and 100 pellets on the second day; the pellets were presented en masse on a plate in their home cages.

Pretreatment. In the pretreatment phase, the animals that were given leverpress training in the Skinner box were randomly assigned to either experimental, yoked, or yoked-signal groups. The experimental group could earn a food pellet by fixed-ratio (FR) 5 leverpresses on the third day of the experiment and by FR 20 on the fourth day, up to 100 pellets each day. The yoked group was yoked to the experimental group; they obtained pellets at the same time as the experimental rats except that the pellet delivery was independent of the yoked animals' behavior. The yoked-signal group was also yoked to the experimental group, but this group ran immediately after the session for the experimental and yoked groups and received response-independent food at the same intervals as the experimental rats. Moreover, the noncontingent food for the yokedsignal group followed a 1.5-sec tone. Thus, the yoked-signal rats received response-independent, predictable food in this phase. The naive control group received 100 pellets per day; the pellets were presented en masse on a plate in their home cages.

Test. On Day 5, all rats were tested with the two-crossing (FR 2) shuttle-escape task. A 0.8-mA shock was delivered until rats crossed the shuttle box twice. If the rats failed to escape within $30 \mathrm{sec}$ after shock onset, the trial automatically terminated and a 30-sec latency was recorded. The intertrial interval had a mean of $40 \mathrm{sec}$, with a range between 20 and $60 \mathrm{sec}$. Thirty trials of the escape task were administered.

\section{RESULTS}

\section{Pretreatment}

Figure 1 shows the mean number of responses across the last 5 min of each schedule (CRF, FR 5, and FR 20). Separate analyses of variance (ANOVAs) conducted on groups for each schedule did not indicate a significant main effect for CRF but revealed significant effects for FR 5 and FR $20[F(2,24)=113.27$ and 67.26, respectively, $p s<.01]$. Subsequent Newman-Keuls tests $(p<$ .05) conducted on the FR 5 and FR 20 schedules revealed that the experimental group differed from the yoked and the yoked-signal groups, which did not differ from each other. Separate repeated measures ANOVAs conducted on schedules revealed significant effects for the experimental, yoked, and yoked-signal groups $[F(2,16)=$ $37.47,55.50$, and 48.21 , respectively, $p s<.01]$.

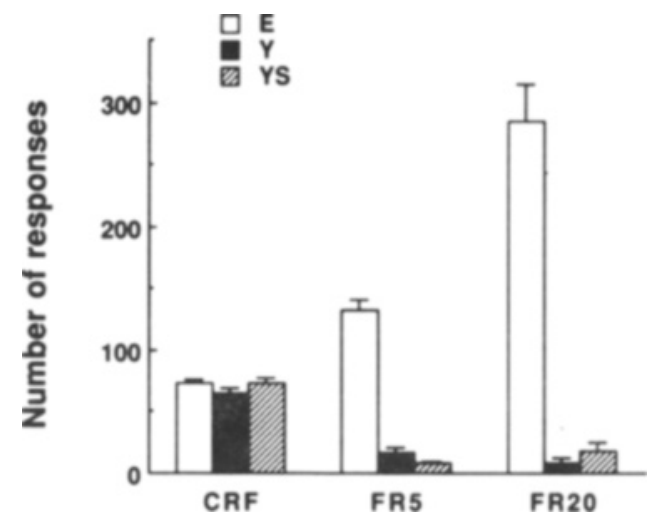

Figure 1. Mean number of responses during the last $5 \mathrm{~min}$ on the CRF, FR 5, and FR 20 sessions for experimental (E), yoked (Y), and yoked-signal (YS) groups. (Vertical lines indicate standard errors.)

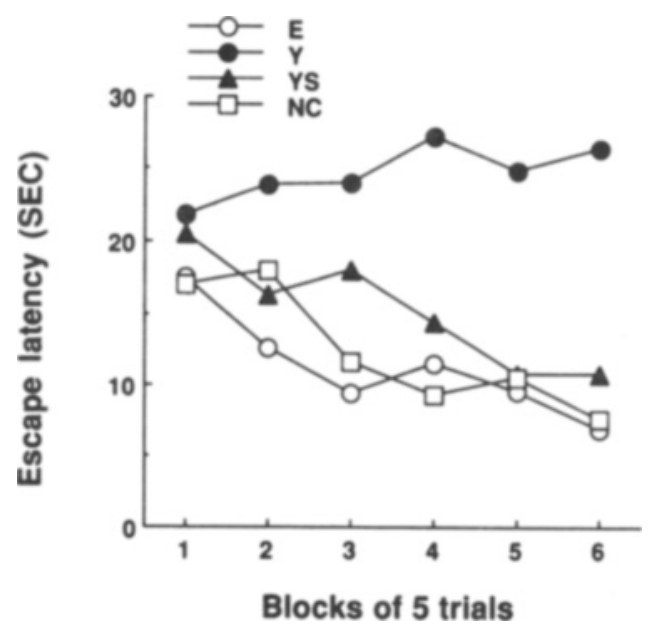

Figure 2. Mean response latencies on the shuttle-escape task for experimental (E), yoked (Y), yoked-signal (YS), and naive control (NC) groups across blocks of five trials.

\section{Test}

Figure 2 shows the mean response latencies on the shuttle-escape task for each group across blocks of five trials. A 4 (groups) $\times 6$ (blocks) two-way ANOVA, with one factor repeated, revealed reliable main effects of groups $[F(3,32)=13.40, p<.01]$ and blocks of five trials $[F(5,160)=5.41, p<.01]$, as well as an interaction effect of groups and blocks $[F(15,160)=2.41$, $p<.01]$. Subsequent Newman-Keuls tests $(p<.01)$ found that the yoked group differed from the experimental, yoked-signal, and naive control groups, which did not differ from each other.

\section{DISCUSSION}

The present experiment showed that addition of a predictive signal of food to the uncontrollable situation 
eliminated the impairment of subsequent escape performance that otherwise resulted from uncontrollability of food acquisition. This modulation effect is analogous to that found for the effects of forward and backward signals of shock conducted in aversive pretreatment situations (e.g., Jackson \& Minor, 1988; Volpicelli et al., 1984).

Many reports indicate the importance of safety signals during sessions in which uncontrollable shocks are presented in preventing subsequent escape deficits (e.g., Minor et al., 1990). However, the present prophylactic effect produced by administration of predictable food during pretreatment cannot be explained in terms of a safety signal because there could be no fear of shock in the present pretreatment phase.

One possible explanation of the prophylactic effects observed in this experiment is based on overshadowing of the instrumental relation by the Pavlovian relation. It has been observed that when reinforced responses produced a signal prior to the reinforcer, there was a reduction in the instrumental responding, and this phenomenon was taken as evidence of overshadowing of the response-reinforcer association by a stimulus-reinforcer association (e.g., Baker, 1990; Pearce \& Hall, 1978; Schachtman \& Reed, 1990). If the differential effect of the predictable signal in the present experiment is due to overshadowing, it is possible that the yoked-signal group will show more persistent responding than the yoked-nonsignal group because the yoked-signal rats pay less attention to the new noncontingent relation between response and food than the yoked rats. Although a difference in response rates between the yoked and the yoked-signal groups did not appear in the pretreatment phase, it remains possible that there was a shift of attention from the response-outcome relation to the signal-outcome relation in the yoked-signal group because that signal-outcome contingent relation is salient compared with the responseoutcome noncontingent relation. Therefore, these rats might not have learned the uncontrollability that produces the adverse effects. However, further examination of this possibility is necessary.

The present results were consistent with the possibility that the prophylactic effect of controllability may be due to the predictability inherent in controllability, such as that yielded by the response-produced proprioceptive feedback (e.g., Mineka et al., 1984). Furthermore, it remains possible that an associative deficit is not responsible for the effect of uncontrollability, but is responsible for the effect of unpredictability. However, some experiments show that control and prediction exert these influences by different mechanisms (e.g., DeCola et al., 1988; Dess et al., 1983; Maier \& Warren, 1988). Although the present experiment did not reveal a clear dissociation between the effects of control and prediction, differential experimental situations will be able to separate the relative contributions of the effect of prior controllability and predictability experience in appetitive situations.

\section{REFERENCES}

BAKER, A. G. (1990). Contextual conditioning during free-operant extinction: Unsignaled, signaled, and backward-signaled noncontingent food. Animal Learning \& Behavior, 18, 59-70.

CASPY, T., \& LUBOW, R. E. (1981). Generality of US preexposure effects: Transfer from food to shock or shock to food with and without the same response requirements. Animal Learning \& Behavior, 9 , 524-532.

DeCola, J. P., Rosellini, R. A., \& Warren, D. A. (1988). A dissociation of the effects of control and prediction. Learning \& Motivation, 19, 269-282.

Dess, N. K. Linwick, D., Patterson, J., Overmier, J. B., \& LeVINE, S. (1983). Immediate and proactive effects of controllability and predictability on plasma cortisol responses to shocks in dogs. Behavioral Neuroscience, 97, 1005-1016.

GoodkIN, F. (1976). Rats learn the relationship between responding and environmental events: An expansion of the learned helplessness hypothesis. Learning \& Motivation, 7, 382-393.

JA'CKSON, R. L., MINOR, T. R. (1988). Effects of signaling inescapable shock on subsequent escape learning: Implications for theories of coping and "learned helplessness." Journal of Experimental Psychology: Animal Behavior Processes, 14, 390-400.

JOB, R. F. S. (1987). Learned helplessness in an appetitive discretetrial T-maze discrimination test. Animal Learning \& Behavior, 15 , 342-346.

JOB, R. F. S. (1988). Interference and facilitation produced by noncontingent reinforcement in the appetitive situation. Animal Learning \& Behavior, 16, 451-460.

JoB, R. F. S. (1989). Response independent food and escape from frustration. In N. W. Bond \& D. A. T. Siddle (Eds.), Psychobiology: Issues and applications (pp. 469-480). Amsterdam: Elsevier NorthHolland.

Maier, S. F., \& Seligman, M. E. P. (1976). Learned helplessness: Theory and evidence. Joumal of Experimental Psychology: General, $105,3-46$.

MaIER, S. F., \& WARren, D. A. (1988). Controllability and safety signals exert dissimilar proactive effects on nociception and escape performance. Journal of Experimental Psychology: Animal Behavior Processes, 14, 18-25.

Mineka, S., Cook, M., \& Miller, S. (1984). Fear conditioned with escapable and inescapable shock: Effects of a feedback stimulus. Journat of Experimental Psychology: Animal Behavior Processes, 10, 307-323.

Minor, T. R., Trauner, M. A., Lee, C., \& Dess, N. K. (1990). Modeling signal features of escape response: Effects of cessation conditioning in "learned helplessness" paradigm. Journal of Experimental Psychology: Animal Behavior Processes, 16, 123-136.

Overmier, J. B., \& Seligman, M. E. P. (1967). Effects of inescapable shock upon subsequent escape and avoidance responding. Journal of Comparative \& Physiological Psychology, 63, 28-33.

Pearce, J. M., \& HALL, G. (1978). Overshadowing the instrumental conditioning of a lever-press response by a more valid predictor of reinforcer. Journal of Experimental Psychology: Animal Behavior Processes, 4, 356-367.

Rosellini, R. A., Warren, D. A., \& DeCola, J. P. (1987). Predictability and controllability: Differential effects upon contextual fear. Learning \& Motivation, 18, 392-420.

Schachtman, T. R., ReED, P. (1990). The role of response-reinforcer correlation in signaled reinforcement effects. Animal Learning \& Behavior, 18, 51-58.

Seligman, M. E. P: (1968). Chronic fear produced by unpredictable electric shock. Journal of Comparative \& Physiological Psychology, 66, 402-411.

Selugman, M. E. P. (1975). Helplessness: On depression, development, and death. San Francisco: W. H. Freeman.

Seligman, M. E. P., \& Maier, S. F. (1967). Failure to escape traumatic shock. Joumal of Experimental Psychology, 74, 1-9.

Seligman, M. E. P., \& MeYer, B. (1970). Chronic fear and ulcers 
in rats as a function of the unpredictability of safety. Journal of Comparative \& Physiological Psychology, 73, 202-207.

Sonoda, A., Hirai, H., \& OKayasu, T. (in press). Effects of loss of control over food acquisition on subsequent disk-pull escape learning. Japanese Psychological Research.

Sonoda, A., OKayasu, T., \& Hirai, H. (1991). Loss of controllability in appetitive situations interferes with subsequent learning in aversive situations. Animal Learning \& Behavior, 19, 270-275.
VolPiCELU, J. R., Ulm, R. R., \& Altenor, A. (1984). Feedback during exposure to inescapable shocks and subsequent shock-escape performance. Learning \& Motivation, 15, 279-286.

(Manuscript received May 18, 1991; revision accepted for publication April 5, 1992.)

\section{Support for Cognitive Science Research National Institute of Mental Health}

The National Institute of Mental Health (NIMH) aims to support a wide range of basic research in the cognitive sciences as part of its effort to expand knowledge of fundamental aspects of human behavior and adaptation.

NIMH encourages proposals for basic research in such areas as perception, memory, knowledge, thinking, learning, language, and performance. Methods may include (but are not limited to) human and animal experimentation, computer simulation, and formal/computational analysis. Investigators may have backgrounds in psychology, computer science, linguistics, philosophy, or any other cognitive science.

Funding mechanisms include grants for research projects (of various sizes and durations), pre- and postdoctoral fellowships, institutional training grants, career scientist awards, and conference grants.

Questions about NIMH's support for cognitive science research can be directed to Rodney Cocking or Howard Kurtzman, Basic Behavioral and Cognitive Sciences Research Branch, National Institute of Mental Health, Room 11C-16, 5600 Fishers Lane, Rockville, MD 20857 (e-mail, kwh@cu.nih.gov; phone, (301) 4439400; FAX, (301)443-4822.) 